\title{
HERRAMIENTAS EN 3D PARA EL MODELADO DE ESCENARIOS VIRTUALES BASADOS EN LOGO. ESTADO DEL ARTE
}

\author{
3D TOOLS FOR MODELING VIRTUAL SCENARIOS BASED ON LOGO. \\ STATE OF THE ART
}

Luz, Santamaría Granados

Ingeniera de Sistemas. Especialista en Telemática. Candidata a Magíster en Ciencias de la Información y las Comunicaciones. Grupo de Investigación GIBRANT, Tunja, Colombia. Docente Facultad de Ingeniería de Sistemas. Universidad Santo Tomás Tunja seccional Tunja.

E-mail: Isantamaria@ustatunja.edu.co

Juan Francisco, Mendoza Moreno

Ingeniero de Sistemas. Especialista en Diseño y Construcción de Soluciones Telemáticas. Grupo de Investigación GIBRANT, Tunja, Colombia. Decano Facultad de Ingeniería de Sistemas. Universidad Santo Tomás seccional Tunja. E-mail: jmendoza@ustatunja.edu.co

Fecha de recepción: 1 de julio de 2009.

Fecha de aprobación: 9 de noviembre de 2009

\section{RESUMEN}

Este artículo revisa la comprobada fundamentación pedagógica de LOGO (Papert, 2003) que a su vez ofrece interesantes estrategias de motivación para los niños, en aspectos tales como el desarrollo de habilidades espaciales a través de su propia exploración de mundos virtuales. La metodología original fue propuesta por Seymour Papert para escenarios en dos dimensiones (2D). Por lo tanto, se analiza la posibilidad de integrar las ventajas pedagógicas de LOGO con una interfaz gráfica en tres dimensiones (3D), al aprovechar la tecnología contemplada en los estándares del consorcio Web3D. Además menciona los componentes X3D que permiten el uso de avatares (humanoides) para facilitar la interacción de los usuarios en mundos virtuales dinámicos, al disponer de personajes adicionales al de la tortuga de LOGO.

Palabras clave: LOGO, humanoide, extensible tridimensional, X3D, SAI, Ajax3D.

\begin{abstract}
This article reviews the tried pedagogical focus of LOGO (Papert, 2003), which also offers interesting motivational strategies for children in areas, such as a spatial skills
\end{abstract}


development from their own exploration of virtual worlds. The original methodology was proposed by Seymour Papert for 2D scenarios. In fact, we analyze the possibility to integrate the LOGO teaching advantages with a 3D graphical interface by leveraging the technology provided by Web3D consortium standards. X3D also describes the components that allow the use of avatars (humanoid) to make easier user interaction in dynamic virtual worlds to count on extra characters of the LOGO turtle.

Keywords: LOGO, humanoid, extensible tridimensional, X3D, SAI, AJAX3D.

\section{INTRODUCCIÓN}

La Realidad Virtual (RV) (Santamaría, 2006) ofrece a los educadores una nueva e innovadora manera de motivar a los estudiantes hacia el aprendizaje de una temática dada, en cualquier área del conocimiento, a través de entornos visuales 3D altamente inmersivos, es decir, que el estudiante tenga la sensación de estar realmente dentro del mundo virtual, y para lograrlo puede utilizar dispositivos como cascos, guantes o lentes para visualizar e interactuar con la aplicación y no simplemente limitarse a consultar páginas web.

Además puede hacer uso de herramientas multimediales o apoyarse con software educativo y experimentar heurísticamente los escenarios virtuales que simulan lugares reales e inalcanzables en tiempo (hechos históricos en el desarrollo de la humanidad) y espacio (museos, laboratorios), que por condiciones económicas, sociales, políticas y culturales no se encuentran al alcance de todas las personas, pero que hoy se logran conocer aunque sea de manera virtual. Para tal propósito existen consorcios en Internet como el Web3D (Web3D Consortium, 2009), considerada como la organización más representativa para crear lenguajes y plataformas de RV, enfocadas en la construcción de aplicaciones virtuales en 3D. Las herramientas y recursos que se comparten tienen la característica de ser interoperables y además de no requerir de algún tipo de licenciamiento.

Las aplicaciones de Diseño Asistido por Computador (CAD) (Visual Aircraft, 2008) están soportando procesos de aprendizaje que involucran conocimiento espacial, tal es el caso de LOGO, un lenguaje de programación que utiliza un enfoque metodológico (Papert, 1987), para facilitar la comunicación del estudiante con el computador basado en el desarrollo del pensamiento concreto al formal, al explorar el ambiente espacial de LOGO para la solución de problemas que están relacionados con destrezas sicomotoras (Wikipedia, 2009).

El artículo integra tres temáticas: Ambientes virtuales de aprendizaje, Herramientas para entornos Web 2.0 y Lenguaje LOGO: 
- La primera hace una reflexión sobre la utilización de la infraestructura tecnológica y las herramientas Web 2.0, como soporte al trabajo colaborativo en redes avanzadas de aprendizaje que tienen la finalidad de compartir recursos y servicios educativos a la comunidad académica e investigativa.

- La segunda menciona los componentes del lenguaje modelado Extensible 3D, para la construcción de escenas virtuales con nodos de script, objetos, sensores de movimiento y ambientación, agrupados en el nodo de escena que provee la interacción con el motor de ejecución y los parámetros de configuración de la simulación, así como el diseño de la estructura de avatares que define los distintos niveles de articulaciones, para proporcionar al usuario final una mayor interacción en la escena virtual. También se relacionan los protocolos y herramientas que hacen parte, tanto del servidor como del cliente, en la arquitectura AJAX, con soporte a la Interfaz de Acceso a la Escena 3D.

- La tercera resalta la importancia de LOGO como herramienta educativa para el desarrollo de competencias espaciales y matemáticas, a partir de la descripción de la metodología propuesta por Seymour Papert (Papert, 1999), a través de un avatar o tortuga que permite crear micromundos en $2 \mathrm{D}$, esta última caracteristica evidencia la posibilidad de trabajar un prototipo de LOGO en 3D para niños con problemas de lateralidad.

\section{AMBIENTES VIRTUALES EN LA WEB 2.0}

En Internet con el auge de las plataformas computacionales distribuidas e inteligentes y las empresas, consorcios e instituciones educativas están haciendo uso de las herramientas que ofrece la Web 2.0 (Fontana F., et al., 2008) (Santamaría y Pérez, 2008) para transferir el conocimiento a través de las redes sociales y compartir recursos de manera colaborativa (Bouras Ch., et al., 2008), por otra parte están surgiendo Redes Avanzadas de Aprendizaje (RAA) como: la Red Nacional Académica de Tecnología Avanzada (RENATA) que se interconecta con la Red Avanzada de Cooperación en Latinoamérica (CLARA), GÉANT en Europa e INTERNET 2 en Estados Unidos (Rendón, 2009), para fortalecer el desarrollo de todas las áreas del saber, al permitir la conformación de grupos de trabajo interdisciplinarios e interinstitucionales con la vinculación de proyectos que benefician el progreso cultural, educativo y económico de la Sociedad del Conocimiento (figura 1). 


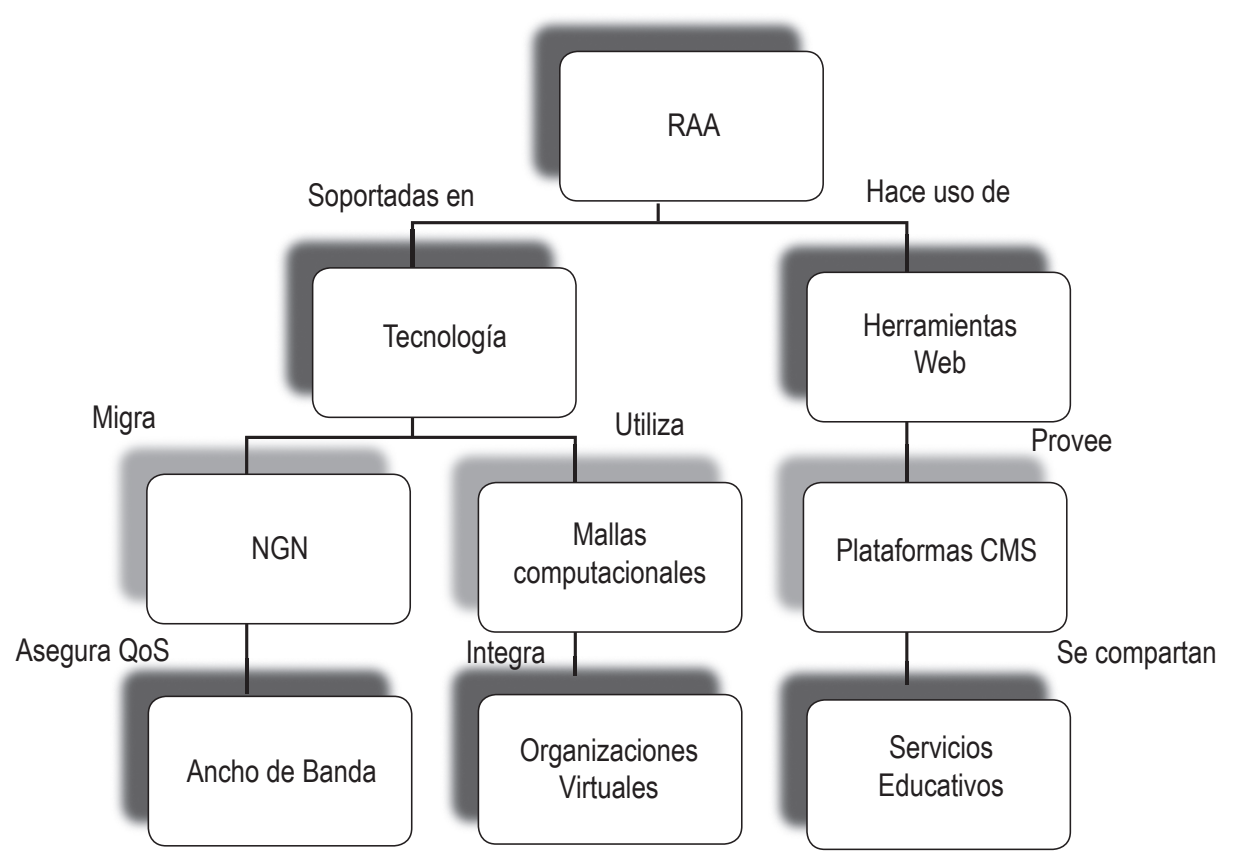

Figura 1. Mapa conceptual Redes Avanzadas de Aprendizaje

En Colombia se ha conformado la iniciativa nacional de computación en malla Grid Colombia (Castro, et al., 2008), para que las universidades y consorcios se conecten a los nodos de la red RENATA, con el fin de publicar productos educativos resultantes de los procesos de investigación, tal como se maneja en una Organización Virtual (OV) que fortalece la calidad académica de las Instituciones vinculadas, al integrar mallas computacionales (grid computing) (Hoyos y Pérez, 2007), para gestionar recursos de procesamiento, almacenamiento y ancho de banda. Una organización virtual inteligente establecida sobre computación distribuida es Second Life (SL) (Linden Lab, 2009), desarrollada por Linden Lab, que crea una versión digital del usuario o 'avatar', para que interactué en tiempo real con objetos virtuales y sitios creados en 3D, que han establecido su propio sistema económico para manejo de las propiedades virtuales de los usuarios y organizaciones.

Como proyecto de colaboración que se ha desarrollado en RENATA se resalta las investigaciones realizadas por la Universidad de los Andes, Universidad Nacional y Universidad de Alberta (FIGUEROA, et al., 2008), al utilizar plataformas de modelado Extensible 3D para la construcción de sistemas de realidad virtual inmersivos, al integrar el diseño de objetos 3D con interfaces hápticas, que ofrece a los usuarios la sensación de tocar físicamente los objetos (analizarlos y limpiarlos), resaltando la importancia de proveer interfaces intuitivas y que provean al usuario realismo total al estar en contacto con una escena virtual, como el caso del museo del oro. 
Por otra parte las plataformas de Sistema de Gestión de Contenidos (Content Management System, CMS) de tipo Open Source como: Drupal, Joomla y Moodle (Michelinakis D., 2004), están siendo adoptadas como solución a las necesidades educativas, pero requieren soporte en la construcción de aplicaciones interactivas que mantengan la motivación de los estudiantes que finalizan los cursos de forma exitosa, alcanzando así el dominio de los conceptos, técnicas o habilidades de aprendizaje, para lo cual se han generado Entornos Virtuales Multi-Usuario (Kemp y Livingstone, 2007) que integran sistemas de gestión de aprendizaje con SL, este sistema híbrido usa la plataforma Moodle y las características de conectividad SL (Sloodle) para diseñar objetos de aprendizaje altamente interactivos que respaldan el proceso de aprendizaje.

\section{HERRAMIENTAS PARA ENTORNOS WEB 3D}

\subsection{COMPONENTE JAVASCRIPT EN X3D}

El Lenguaje Extensible Tridimensional (X3D) (Consortium, 2008), se considera como la evolución del Lenguaje Modelado de Realidad Virtual (Virtual Reality Model Lenguaje, VRML) y se caracteriza por implementar aplicaciones interactivas en 3D, que integran recursos multimediales, dispositivos 3D y arquitectura basada en componentes, esta última puntualiza la diferencia con VRML debido a que realiza un diseño modular más estructurado para definir la ambientación de la escena con los componentes de sonido, iluminación y efectos; y para el diseño de los objetos dispone de los componentes de geometría, renderizado y comportamientos que genera el aspecto dinámico en los mundos virtuales, y que para tal propósito el Web3D definió los componentes de: programación en javascript, sensores e interpolación de movimiento.

Para construir una escena en X3D se parte de la agrupación jerárquica de nodos que permiten definir características y relaciones de los objetos a partir del nodo Escena (Scene) (Web3D Consortium, 2009), donde se definen los nodos de intercambio para grupos de objetos (geometría o indexedFaceSet, textura o ImageTexture y animación o Collision) que facilitan el acceso a aplicaciones Web; los nodos de interacción (importar archivos o inLine, terreno regular o ElevationGrid, eventos al hacer clic o TouchSensor) programan acciones del usuario a través de sensores; por último los nodos de inmersión (Script, neblina o Fog, sonido o Sound) utilizan programación en scripting y configuran la ambientación de la escena para hacerla lo más real posible (figura 2).

En una escena virtual el motor de ejecución (figura 3) recibe los parámetros de la animación (eventos de salida y campos) que provienen del nodo Scene (nodos de objetos, sensores y script), para ser configurados como eventos que tienen el mismo tipo de dato en la ruta (ROUTE) de conexión entre dos nodos. Los eventos de entrada, salida y campos en el ROUTE definen el comportamiento interactivo que se programa cuando 


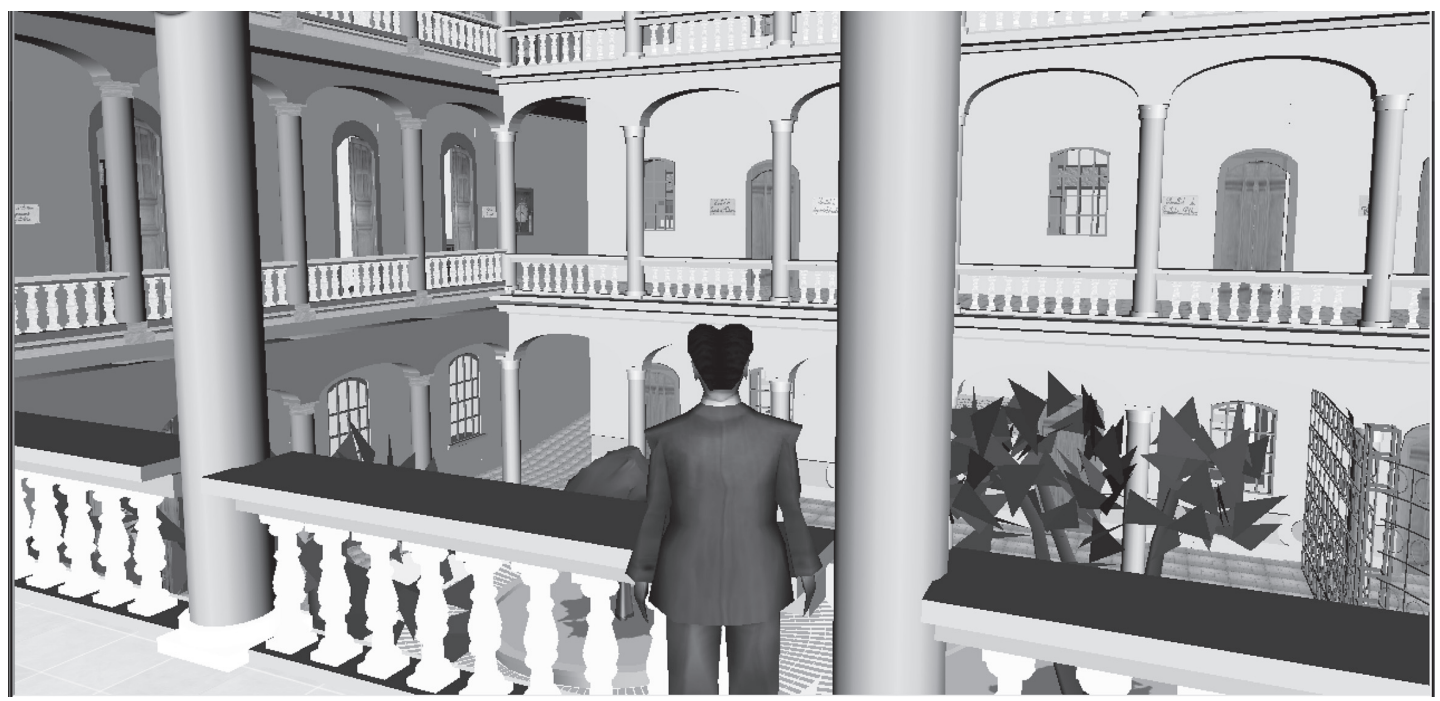

Figura 2. Modelo virtual Universidad Santo Tomás de Tunja.

se incluye un nodo Script, por otra parte en los eventos del ROUTE se obtiene información de los nodos de la escena que se ejecutarán en un instante de tiempo (nodo TimeSensor) de acuerdo al proceso de la animación.

El nodo Script para el control de eventos define los tipos de datos en el elemento field y los relaciona dependiendo de los eventos recibidos o enviados por el ROUTE, en la figura 4 se estructuran jerárquicamente los nodos y campos relacionados en la animación del nodo Script. Por otra parte se ha evidenciado el desarrollo de modelos de simuladores (Jiménez M., et al., 2005) (González A., et al., 2005) que controlan las funciones de robots con cálculos y funciones específicas, basándose en la configuración de eventos para ser ejecutados en un ciclo de tiempo definido a través del sensor de tiempo, la función de los eventos se especifica a continuación:

- Eventos de entrada: debe ser igual al nombre de la función en el script y define el evento de salida del script en el ROUTE.

- Eventos de salida: genera el valor que debe enviarse al objeto 3D para modificar el comportamiento en la escena. Define el evento de entrada en el ROUTE.

- Campo: se utiliza como parámetro para hacer cálculos en la función script.

- ExposedField: integra la funcionalidad de los tres anteriores.

Por otra parte para el soporte de tipos de datos en los campos en X3D se configuran como objetos ECMAScript (ECMAScript, 2007) y se pasan por referencia, los tipos de datos que se definen como booleano (bool), entero (int32), doble (double), decimal (float) o cadena de caracteres (String). El campo SFBool se representa con valores constantes en TRUE o FALSE. Los campos SFInt32, SFFloat y SFTime son configura- 


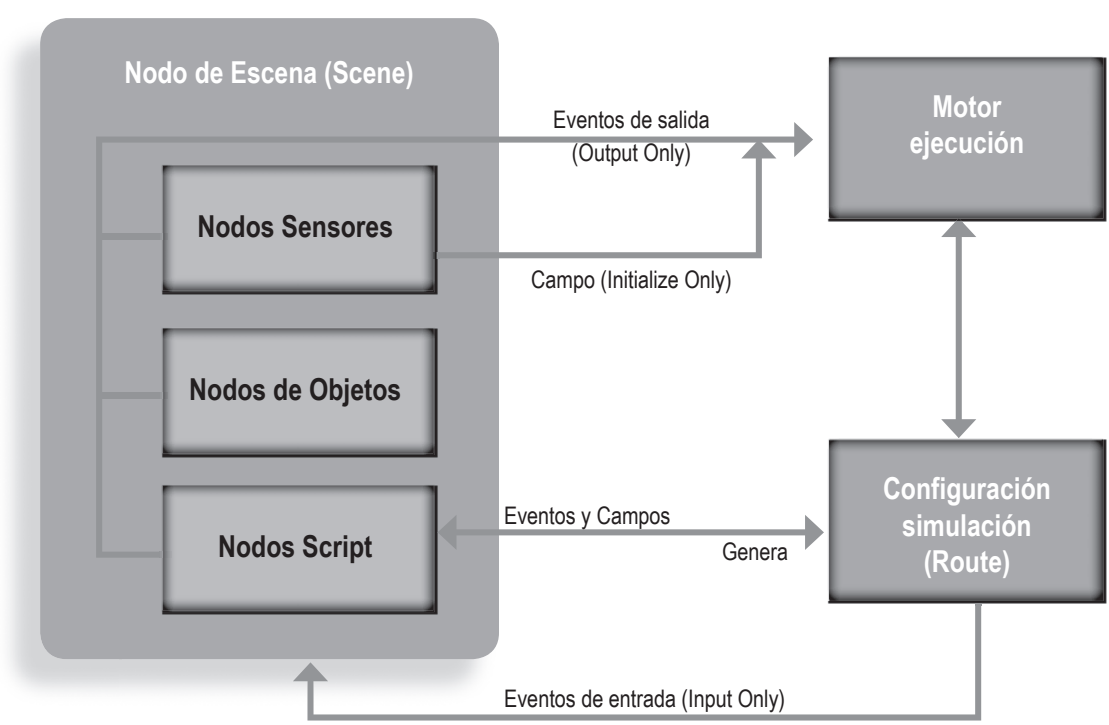

Figura 3. Esquema de animación de la escena virtual (VideaLab, 2008)

dos como numéricos. Estos tipos de datos se utilizan como valores dentro del llamado de las funciones y se agrupan en:

- Los tipos de datos SF: el tipo de dato debe ser el correspondiente para el objeto SF (vector).

- $\quad$ Los tipos de datos MF: se asignan a un elemento de un objeto MF para una variable de intervalo (matriz) que crea una referencia a ese elemento.

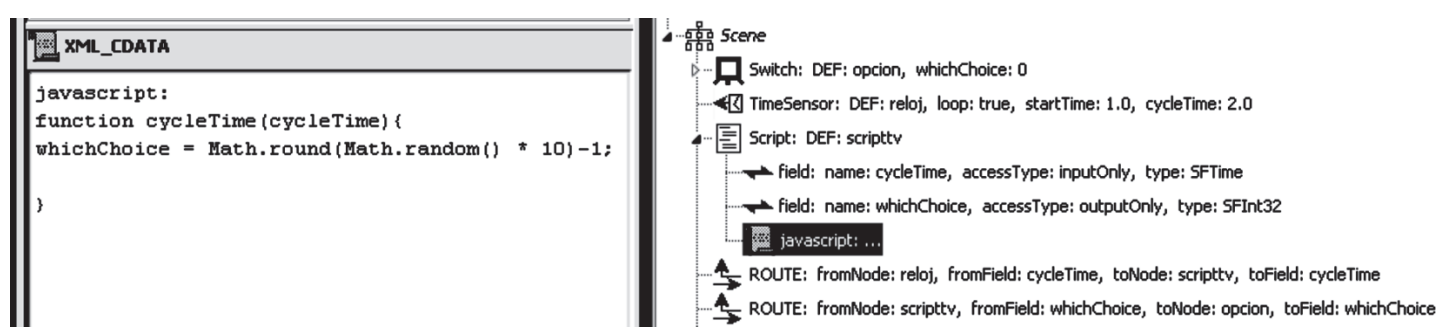

Figura 4. Descripción del nodo Scene con definición de eventos en X3D-Edit

\subsection{MODELADO DE AVATAR}

En el diseño del humanoide se integran lenguajes de animación de scripting y herramientas de modelado visual, el primero describe las tareas que debe hacer el avatar, definidas en su nivel de detalle o complejidad en los comportamientos de interacción en el mundo virtual y el segundo facilita el diseño geométrico con herramientas de extrusión que crean formas 2D para luego ser proyectadas en 3D; por otra parte los po- 
lígonos del humanoide pueden ser perfeccionados con IndexedFaceSet (IFS). Existen prototipos de herramientas implementadas para el modelado de humanoides con X3D como H-Animator (Buttussi y Chittaro, 2006), que reutiliza y comparte la configuración de la animación y modelado en 3D a través de una base de datos.

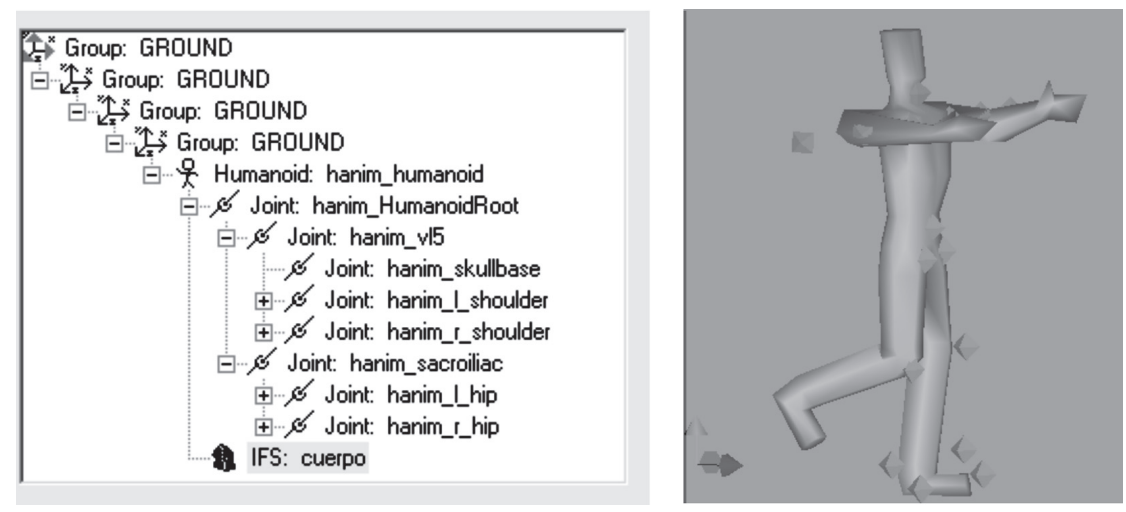

Figura 5. Especificación nodos del componentes H-Anim.

Por otra parte se cuenta con herramientas comerciales como VizX3D (Virtlock Technologies, 2005) y Vivaty Studio (Vivaty, 2009) que facilitan la construcción del humanoide y la simulación e interacción del avatar con los objetos 3D en el escenario virtual. En la figura 5 se muestra la estructura del humanoide, que se encuentra estandarizada a través del componente H-Anim (Web3D Consortium, 2008) que intregra los nodos básicos joint y segment y los nodos avanzados site y displacers:

- $\quad$ El nodo H-Anim se emplea como la raíz (root) del avatar y contiene información general del avatar.

- $\quad$ Nodos Joint son los nodos de transformación y de rotación que dan vida al avatar.

- $\quad$ Nodo Segment contiene los nodos de la geometría.

- $\quad$ Nodo Site especifica los sitios de interés, como extremidades del dedo, y las cejas.

- $\quad$ Nodo Displacer simula acciones de los músculos que no se asocian con los nodos Joins como el movimiento de las cejas, labios y respiración.

Jerárquicamente los nodos coordinan la rotación y desplazamiento del avatar por medio de los nodos Joint VL5 que integra las extremidades superiores desde la base del cráneo (skullbase), hombros (shoulder), codo (elbow) y muñeca (wrist) tanto para la parte derecha como para la izquierda. Las extremidades inferiores se controlan con el nodo Joint Sacroiliac conformado por la cadera (hip), rodillas (knee), tobillos (ankle) y pie (midtarsal) de igual forma para los dos laterales.

En el modelado del humanoide se puede especificar tres Niveles de Articulación (Levels of Articulation, LOA) que conforman la estructura del esqueleto (Silverglate, 2006): 
LOA1 se caracteriza por ser un modelo básico ya que integra 18 nodos Joins y no incluye diseño del rostro, dedos y la columna vertebral es muy rígida. LOA2 maneja 71 vertebras con articulaciones en las manos, pero no en la cara y finalmente el modelo LOA3 es el más completo porque adhiere 89 nodos Joins para los movimientos de la columna vertebral, manos y rostro. La animación del humanoide se soporta en el componente de sensores de interpolación (Web3D Consortium, 2008) como el nodo PositionInterpolator que controla el desplazamiento del humanoide y el OrientationInterpolator que define la rotación de las articulaciones del avatar a través de fotogramas definidos en intervalos de tiempo en segundos y que se configura en el nodo de animación (Animated Node).

Para Ambientes Virtuales en Red (Networked Virtual Environments, NVEs) (Bouras et al., 2009) (Suárez Quirós, et al., 2003) la colaboración entre los usuarios se soporta en arquitecturas Cliente/Servidor (C/S) que integran un servidor Web (Apache, TomCat) junto con herramientas de desarrollo (PHP - Preprocesador de Hyper Texto, Java) para controlar los procesos de interacción de la escena con el servidor de bases de datos (MySQL, Postgres, Oracle), que permite almacenar y modificar los datos de los eventos, y que el cliente a través del avatar explora e interactúa con otros usuarios. La herramienta BS-Colaborate (Bitmanagement Software $\mathrm{GmbH}, 2007$ ) ofrece un sistema colaborativo en 3D que soporta el estándar X3D, para la construcción de objetos 3D en ambientes multiusuario con contenido educativo o de entretenimiento, para tal propósito ha incluido nodos especializados para el manejo de sesiones y eventos de los usuarios en la conexión a la escena virtual.

\subsection{AJAX 3D}

Los servicios Web se caracterizan por ser ágiles y dinámico al integrar AJAX (Asynchronous JavaScript And XML, Javascript y XML Asíncronos) (Eguíluz Pérez, 2008) en el diseño de interfaces de usuario. La figura 6 muestra el esquema de funcionamiento de la arquitectura C/S que soporta la transferencia de datos en el Lenguaje de Marcas Extensible (Extensible Markup Language, XML), al permitir modificar la información en tiempo real sin tener que recargar las páginas en el navegador. AJAX (Eguíluz Pérez, 2008) está conformado por tecnologías de Modelo de Objetos del Documento (Document Object Model, DOM) y Lenguaje Extensible de Marcado de Hipertexto (eXtensible Hypertext Markup Language, XHTML); el DOM cuenta con un conjunto de utilidades específicamente diseñadas para manipular documentos XML que manejan un repositorio de funciones de Interfaz de Programación de Aplicación (Application Programming Interface, API) (W3C, 2007), estas funciones son utilizadas en distintos entornos Web para manipular de formar rápida y eficiente las páginas XHTML.

El diseño de aplicaciones y portales esta soportado en lenguajes de programación como JavaScript y Java. DOM tiene la finalidad de facilitar la portabilidad entre los 


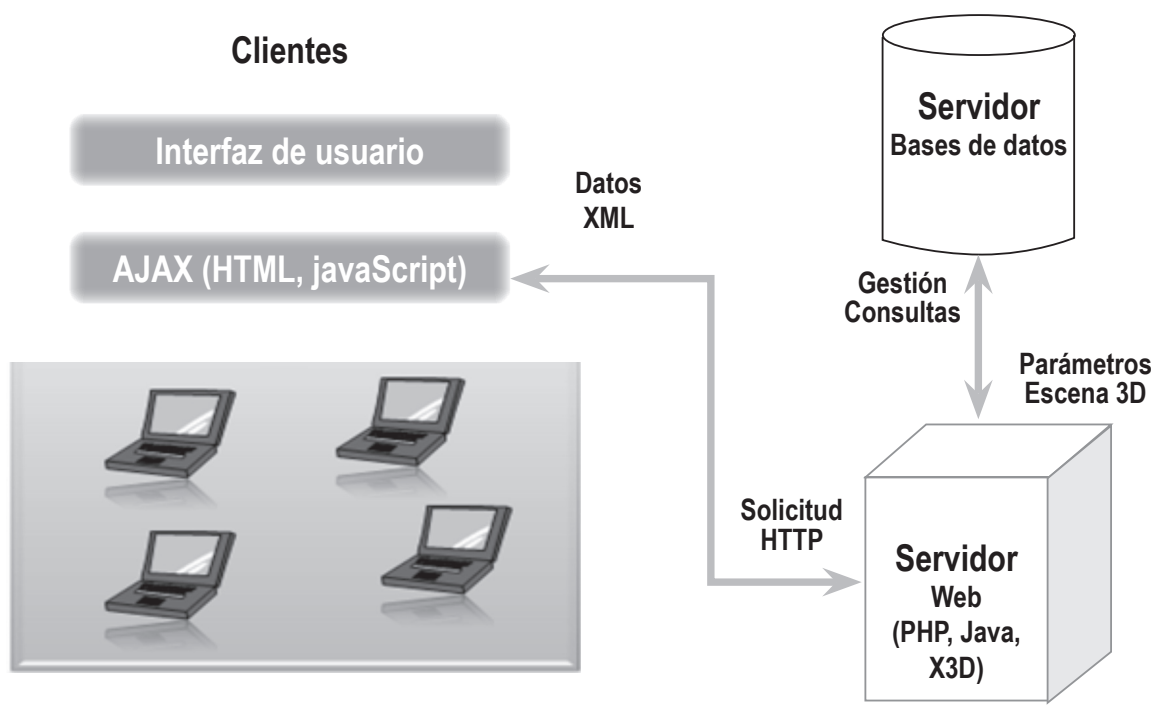

Figura 6. Arquitectura AJAX

navegadores Web. AJAX para el diseño de las páginas Web emplea la especificación de Hojas de Estilo (Cascade StyleSheet, CSS) (W3C, 2009), este tiene como propósito aplicar estilos a la presentación de la estructura lógica del documento HTML. Aprovechando la interoperabilidad de los estándares del Web3D y del WWW: AJAX y X3D para el desarrollo de aplicaciones Web 3D, Toni Parisi (Parisi, 2006) los integró y propuso AJAX3D, para facilitar el acceso al mundo virtual a través del componente de Interfaz de Acceso a la Escena (Scene Access Interface, SAI) (Web3D, 2008) (Santamaría y Peréz, 2008), que incorpora JavaScript para configurar los parámetros de entrada al control del entorno 3D, que cualquier usuario puede visualizar con un plugin que instala en su computador.

Para comunicarse con el servidor, DOM cambia la página Web en el proceso de intercambio de información 3D generado por el método "XMLHttpRequest" y SAI, para el control de solicitudes en tiempo real a la escena 3D utiliza el método "createX3DFromURL". Estos métodos son usados por el servidor para almacenar y recuperar información de la escena en X3D.

\subsubsection{Requerimientos para una escena virtual con Ajax3D.}

El Web3D en el desarrollo de la tercera fase para generar aplicaciones 3D en Internet (Bullard, 2007) ofrece la compatibilidad entre el modelo de interfaz 3D y el DOM de HTML, para que la escena virtual pueda ser modificada a través de contenidos programados en la página Web. Los usuarios visualizan la escena 3D con Vivaty Studio (Parisi, 2006) que soporta el manejo de la interfaz del cliente SAI, desde donde se conecta con la escena virtual para realizar la construcción o modificación de la misma 
de acuerdo a los parámetros de entrada. Inicialmente en la página HTML incluye el archivo X3D en la etiqueta "EMBED".

El archivo javascript a través del objeto "getBrowser" verifica que el plugin instalado en el navegador soporta SAI, para posteriormente modificar las propiedades y eventos de la escena virtual con el objeto "getExecutionContext". SAI ofrece varias formas de acceder a la escena gráfica a través del nombre del objeto o por una colección de todos los nodos de nivel superior en la escena. En X3D el nodo se considera como el elemento gráfico de la escena, que jerárquicamente representa los nodos de agrupamiento, de formas geométricas, apariencia, y secuencias de comandos que contienen comportamientos e interacciones.

\section{LENGUAJE LOGO}

El lenguaje de programación "Logo" se fundamenta en el aprendizaje constructivo y proporciona herramientas que facilitan el desarrollo de destrezas en lógica espacial y refuerzo de fundamentos matemáticos (Torres M., 1990) para la solución de problemas a través de algoritmos. En la actualidad existen muchas versiones desarrolladas de Logo soportadas en ambientes multiusuario y de aprendizaje colaborativo.

Logo permite construir programas para ejecutarse en el computador, estos programas están conformados por una secuencia de instrucciones que el computador interpreta. Se puede escribir programas relacionados con cálculos matemáticos, diseño de gráficos y formas. Logo funciona como un lenguaje de programación de alto nivel, por lo tanto utiliza un intérprete para ejecutar las instrucciones, denominadas "primitivas" (Muller, 1998).

Se están desarrollando prototipos de LOGO que incluyen manejo de un Lenguaje Orientado a Objetos Natural (NOOP, Natural Object-Oriented Programming) (Boytchev, 2006), para lo cual incluye una biblioteca orientada a la geometría en 3D, que se propone como una pequeña demostración para el pensamiento y aprendizaje natural que ofrece LOGO en la construcción de objetos simples en la práctica del aprendizaje en la programación. Por otra parte, en la Facultad de Tecnología y Cognición de la Universidad del Norte de Texas, se propuso la implementación de una herramienta de LOGO en 3D (Jones y Hicks, 2004) basado en la Biblioteca Grafica Abierta (OpenGL, Open Graphics Library) (OpenGL, 2009), en el momento el módulo de expansión se encuentra en fase de desarrollo.

\subsection{METODOLOGÍA}

Las aplicaciones de CAD están soportando procesos de aprendizaje que involucran conocimiento espacial, tal es el caso de LOGO, un lenguaje de programación que utiliza 
un enfoque pedagógico que ofrece las características necesarias para que el estudiante entienda las tareas que debe realizar en su proceso de aprendizaje (Valente, 1997) (Papert, 1999) y que el docente orienta en el enfoque de enseñanza de la comprensión (Andes, 2009) con criterios que faciliten el desempeño flexible en cualquier tema que se conoce. LOGO ofrece un entorno para que los estudiantes apliquen diversas estrategias en la solución de problemas, pero esta interacción debe estar mediada por un tutor o docente. La metodología de LOGO se enfoca en tres aspectos:

1. Interacción con el computador: Para Seymour Papert (Papert, 1999) comunicarse con el computador facilita la forma de producción del aprendizaje, a través de la programación en LOGO, ya que los estudiantes al conocer algunas instrucciones de LOGO como el desplazamiento, el giro, el trazado de líneas y de dibujos, se pueden agrupar de manera ordenada en procedimientos para solucionar un problema propuesto.
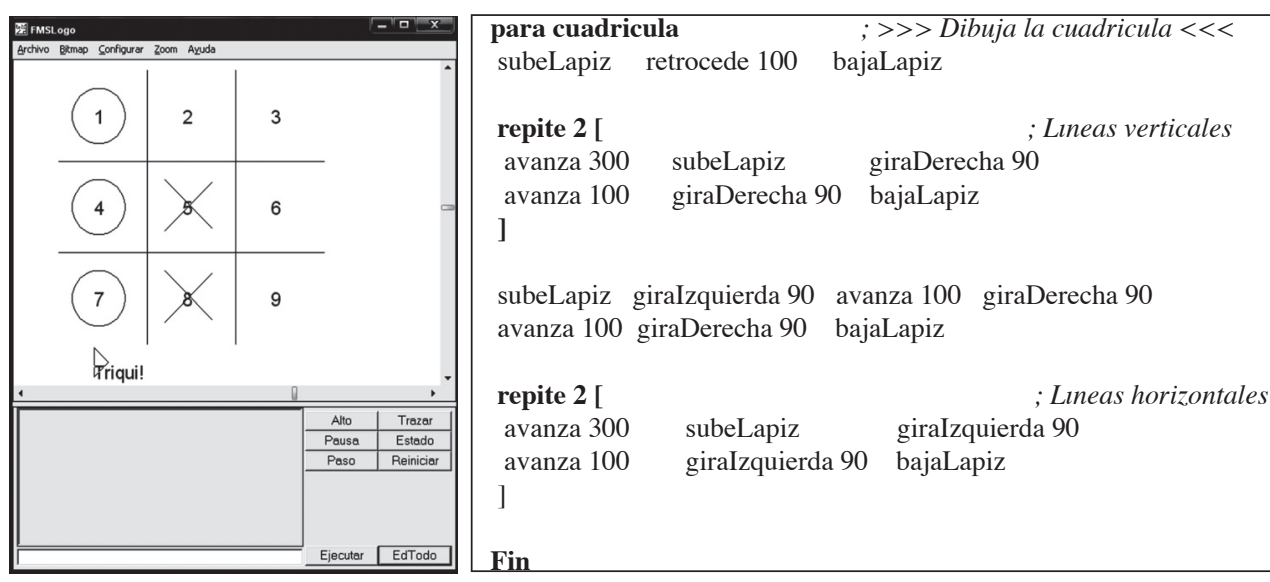

repite 2 [

avanza 300

avanza 100

]

Fin

; Lineas horizontales

subeLapiz giraIzquierda 90

giraIzquierda 90 bajaLapiz

Figura 7. Interfaz de LOGO

La figura 7 muestra la interfaz del ambiente de programación educativo FMSLogo (FMSLogo, 2009), que incluye el diseño gráfico y el conjunto de instrucciones de un procedimiento que hace el trazado de una cuadricula del juego "triqui" (tic tac toe), estos comandos son muy intuitivos para el usuario, por ejemplo el movimiento del lápiz (subir, retroceder, bajar, borrar, avanzar, girar a la izquierda y a la derecha). Los comandos se pueden agrupan en ciclos repetitivos para optimizar la implementación de las aplicaciones que el estudiante realiza con ingenio y haciendo uso del dominio del tema propone soluciones.

2. Pensamiento formal: Papert (Papert, 1999) basado en Piaget (Valente, 1997) en el postulado del desarrollo del pensamiento concreto al formal, hace uso de LOGO como herramienta para mediar este proceso de aprendizaje a través de un docente, quien de acuerdo a las competencias cognitivas y sicomotoras que desee que 
el estudiante genere, define el nivel de complejidad a través de actividades didácticas y lúdicas. El ejemplo de la figura 6 muestra la aplicabilidad del pensamiento formal, el estudiante "juega" con LOGO en un mundo exploratorio educativo, personifica a una tortuga (avatar) que mediante comandos la puede desplazar dentro del mundo virtual, en segundo plano, dibuja elementos geométricos generados por actividades experimentales. Es así como el LOGO posibilita la generación de alternativas distintas para resolver problemas.

3. Evaluación: se considera como parte final del proceso de aprendizaje, porque determina si el estudiante cumplió con los objetivos propuestos, que le posibilitaron adquirir conocimiento espacial, matemático y lógico, para lo cual los criterios de desempeño de compresión (Esteban P., et al., 2004) deben evaluarse de forma continua y en trabajo individual o grupal que motive al desarrollo de competencias comunicativas y cognitivas de cada estudiante.

\section{CONCLUSIONES Y TRABAJO FUTURO}

El desarrollo de aplicaciones y software educativo en ambientes virtuales se caracteriza por ser descentralizado y colaborativo (Badilla S., 2007), para lo cual involucra la creación de sistemas de simulación (Fuentes N. y Herrera, 2001) que soportan los procesos educativos de los distintos niveles de escolaridad, e integra el constructivismo y la tecnología computacional que pretende aplicar estrategias pedagógicas para facilitar el desarrollo de habilidades cognitivas y sicomotoras (Rexach y Asinstein, 1998). La anterior afirmación puede servir como base para proyectos de investigación donde se conjuguen: las ventajas pedagógicas del constructivismo propuestas por Jean Peaget que retomó Papert y las de la realidad virtual no inmersiva, tal como se mencionó en la arquitectura AJAX que provea al usuario la interfaz de acceso a la escena y el repositorio de objetos X3D, para la construcción de mundos virtuales que refuercen el desarrollo de destrezas espaciales y aprendizaje de áreas disciplinares.

Se evidencia la necesidad de desarrollar aplicaciones en 3D que soporten los procesos de entrenamiento en áreas disciplinares como: la medicina, la biología, las matemáticas, la física, entre otras, y que la Academia Nacional de Ingeniería de los Estados Unidos (National Academy of engineering, NAE) (NAE, 2009), propone en el desafío de realidad virtual para los Ingenieros de Sistemas. La realidad virtual inmersiva hace uso de componentes tanto de hardware (dispositivos 3D) como de software (herramientas de modelado), se podría plantear el diseño de un prototipo de LOGO inmersivo, por lo tanto se tendrían que realizar las pruebas de compatibilidad de X3D con dispositivos específicos para tal fin.

De los proyectos relacionados con prototipos de LOGO 3D (Boytchev, 2006) (Jones y Hicks, 2004), se puede mencionar que son propuestas investigativas de las que no se 
conocen evidencias como producto terminado, por lo tanto se puede proponer un proyecto investigativo que aproveche el uso de plataformas Web3D como X3D y se centre en el desarrollo de competencias espaciales especificas como la lateralidad, cálculos matématicos y trigonométricos para la construcción de escenarios en 3D. El proyecto de diseño de radio enlaces (Santamaría y Peréz, 2008) está enfocado a ser no inmersivo, pero de igual forma se enfoca al desarrollo de competencias cognitivas específicas.

Al conocer las herramientas de modelado de realidad virtual y la fundamentación pedagógica de LOGO se propone el diseño de aplicaciones virtuales dirigidas a niños que tengan problemas al desarrollar destrezas sicomotoras de ubicación espacial como: la lateralidad, la rotación, la translación, la dimensión y la construcción de objetos y escenarios promovidos por una "tortuguita" (turtle) que es el avatar en el mundo virtual, con el componente h-anim y script se pueden diseñar personajes que estimulen la creatividad de los niños en la creación de los escenarios. De acuerdo con la filosofía del lenguaje de programación LOGO (Pantoja V., 2001) (MIT, 2003), y con el enfoque constructivista que permita motivar el aprendizaje en los niños mientras ellos mismos exploran los escenarios tridimensionales (CINTED, 2007).

\section{REFERENCIAS BIBLIOGRÁFICAS}

[1] Andes. (2009). Enseñanza para la comprensión. Consultado el 5 de 4 de 2009. En: http://learnweb.harvard.edu/andes/tfu/info3d.cfm

[2] Badilla Saxe, E. (2007). Descentralizar el aprendizaje: nuevos retos para la educación. Actualidades Investigativas en Educación , 7, 1-27.

[3] Bouras, C., Chatziprimou, K., Triglianos, V., Tsiatsos, T. (2009). International Conference on Computer Graphics Theory and Applications. Lisboa.

[4] Bouras, C., Giannaka, E., Thrasyvoulos, T. (2009). A framework model for DVEs using SIMUL8. ICST, 1-8.

[5] Bouras, C., Giannaka, E., Thrasyvoulos, T. (2008). E-Collaboration Concepts, Systems and Applications. Encyclopedia of Internet Technologies and Applications, 165-171.

[6] Boytchev, P. (2006). Technology Enhanced Natural Learning. CIST , 1-7.

[7] Bullard, L. (2007). AJAXing The X3D Sequencer. Consultado el 2 de 5 de 2009. En: http://3donthewebcheap.blogspot.com/2007/04/ajaxing-x3d-sequencer.html

[8] Buttussi, F., Chittaro, L. (2006). International Conference on Computer Graphics Theory and Applications. H-Animator: A Visual Tool for Modeling, Reuse and Sharing of X3D Humanoid Animations, (págs. 1-9). New York. 
[9] Castro, Harold. CHACÓN, Jorge. DÍAZ, César. GONZÁLEZ, Enrique. ZULUAGA, Jorge. (2009). Grid Colombia: soporte para investigaciones de avanzada. Encuentro internacional de e-ciencia y educación apoyadas por redes de tecnología avanzada, Universidad ICESI. En: http://www.icesi.edu.co.

[10] Cinted. (2007). Developing a Multiplayer Online Learning Environment to Web Support with AJAX3D and Virtual Reality. Consultado el 27 de 2 de 2009. En: http:// www.cinted.ufrgs.br/ciclo10/artigos/2fEverton.pdf

[11] Ecma Script. (2007). Proposed ECMAScript 4th Edition - Language Overview. Consultado el 15 de 2 de 2009. En: http://www.ecmascript.org/es4/spec/overview.pdf

[12] Eguíluz Pérez, J. (2008). Introducción a AJAX. Bajo Licencia Creative Commons Reconocimiento - No Comercial.

[13] Esteban, P., Restrepo, J., Trefftz, H., Jaramillo, J., Alvarez, N. (2004). VII Congreso Colombiano de Informática Educativa . La Enseñanza para la Comprensión apoyada con la Realidad Aumentada en el Cálculo de Varias Variables. Brasil.

[14] Figueroa, Pablo. Londoño, Eduardo. Prieto, Flavio. Boulanger, Pierre. Borda, Juan. Restrepo, Diego. (2008). Experiencias virtuales con piezas del museo del oro. En: www.renata.edu.col.

[15] Fmslogo. (2009). FMSLogo: An Educational Programming Environment. Consultado el 23 de 2 de 2009. En: http://www.softronix.com/

[16] Fontana, F., Moreno, A., Cosimi, E. (2008). Proceedings of World Conference on Educational Multimedia, Hypermedia and Telecommunications 2008. Web 2.0 Dynamic Virtual Environment to Manage e-Learning Objects and Videos for Synchronous and Asynchronous Lectures and Seminars (págs. 1900-1906).

[17] Fuentes Navarro, R., Herrera, S. (2001). Tecnología, cognición y aprendizaje: construcción educativa de realidades mediante la simulación computacional. ITESO, 1-11.

[18] Gmbh, B. S. (2007). BS Collaborate. Consultado el 5 de 4 de 2009. En: www.bitmanagement.de

[19] González Aguilera, D., Gómez Lahoz, J., Arias Pérez, B. (2005). Consideraciones acerca de GeoVRML: la cartografía 3D en la Web. Mapping Interactivo Revista Internacional de Ciencias de la Tierra , 1-10.

[20] Hoyos Pineda, J. G., Pérez Castillo, J. N. (2007). Aplicación de algoritmos genéticos a la clasificación de imágenes de satélite en el marco de los servicios grid inteligentes. Ciencia e Ingeniería Neogranadina, Vol. 17-2, , 95-109. 
[21] Inc, Virtlock Technologies. (2005). VizX3D. Consultado el 01 de 04 de 2009. En: http://www.vizx3d.com/

[22] Jiménez Macías, E., Moreno Fernández, J. A., Pérez, M. (2005). Congreso XXVI Jornadas de Automática. Simulación en internet de laboratorio virtual en su entorno mediante VRML (págs. 1-6). Alicante-Elche: Universidad de La Rioja.

[23] Jones, G., Hicks, J. (2004). Logo goes 3D. College of Education University of North Texas, 1-4.

[24] Kemp, J., Livingstone, D. (2007). Putting a second life "metaverse" skin on learning management systems. Consultado el 10 de 2 de 2009. En: http://www.sloodle.com/whitepaper.pdf

[25] Lab, Linden. (2009). Second Life Grid. Consultada en: 10 de 3 de 2009. En: http:// secondlife.com/businesseducation/

[26] Michelinakis, D. (2004). Open Source Content Management Systems: An Argumentative Approach. TM Open Source, 1-113.

[27] Mit. (2003). Logo Foundation. Consultado el 24 de 3 de 2009. En: http://el.media. mit.edu/logo-foundation/

[28] Muller, J. (1998). The Great logo Adventure. Massachusetts: Doone Publications.

[29] National Academy of engineering (NAE). (2009). Grand challenges for engineering. Consultado el 19 de 2 de 2009. En: http://www.nae.edu/nae/naehome.nsf

[30] Oecd. (2008). Convergence and Next Generation Networks. Ministerial Background Report , 1-63.

[31] Open GL. (2009). Open GL Overview. Consultado el 18 de 2 de 2009. En: http:// www.opengl.org/

[32] Pantoja Vallejo, A. (2001). Influencia del lenguaje de programación Logo en la capacidad creativa del niño del Tercer Ciclo de Educación Primaria. Buenos Aires: Servicio de publicaciones de la Universidad de Jaén.

[33] Papert, Seymour. (1987). Desafío A La Mente. computadoras y Educación. Buenos Aires: Galápagos.

[34] Papert, Seymour. (29 de 03 de 1999). Papert on Piaget. Time magazine, pág. 105. 
[35] Papert, Seymour. (2003). Portal de Seymour Papert. Consultado el 19 de 3 de 2009. En: www.papert.org/

[36] Parisi, Tony. (2006). Ajax3D: The Open Platform for Rich 3D Web Applications. Consultado el 10 de 2 de 2009. En: AjaX3D: http://www.ajax3d.org/whitepaper/

[37] Rendón, H. (2009). Rumbo - Renata - Clara: Un Camino hacia la Ciencia Redes Académicas Avanzadas de Alta Velocidad. Consultado el 1 de 6 de 2009. En: http://www.rumbo.edu.co/file.php/1/info/

[38] Rexach, V., Asinstein, J. (1998). Yendo de la tortuga al mouse. Buenos Aires: Novedades Educativas.

[39] Santamaría Granados, L. (2006). Simbiosis de realidad virtual y educación. Revista Educación y Ciencia, ISSN 01207105, 19-24.

[40] Santamaría Granados, L., Perez Castillo, J. N. (2008). Laboratorio virtual para el diseño de radio enlaces en un ambiente grid. Ciencia e Ingeniería Neogranadina, $18(2), 61-76$.

[41] Santamaría Granados, L., Perez Castillo, J. N. (2008). Laboratorios virtuales sobre mallas computacionales como soporte al aprendizaje colaborativo en el área de GIS\&T. Revista Umbral Científico, Número 13. Segundo semestre, 87-95.

[42] Silverglate, D. (2006). Tutorial Humanoid Animation. Chief Software Developer Vcom3D, Inc. Consultado el 15 de 3 de 2009. En: http://www.web3d2006.org/slides/H-Anim-2006.pdf

[43] Suárez Quirós, J., García, R. R., Gallego Santos, R., González, S. M. (2003). Desarrollo de un entrenador para la percepción espacial basado en realidad virtual mediante tecnologías de dominio público. Consultado el 10 de 3 de 2009. En: http://www.upc.es/euetib/xiicuieet/comunicaciones/din/comunicacions/5.pdf

[44] Torres M., C. (1990). Logo: una nueva respuesta para la enseñanza de los primeros conceptos en matemáticas. Consultado el 13 de 3 de 2009. En: http://www. colombiaaprende.edu.co/html/mediateca/1607/articles-127144_archivo.pdf

[45] Valente, J. A. (1997). La función de los ordenadores en la educación: destreza y comprensión. Perspectivas, XXVII (3), 433-446.

[46] Videalab. (2008). Interacción en 3D. Consultado el 5 de 3 de 2009. En: http://videalab.udc.es/i3d/ 
[47] Visual Aircraft. (2008). The X3D CAD Initiative. Consultado el 2 de 5 de 2009. En: http://visualaircraft.com/the-x3d-cad-initiative/

[48] Vivaty. (2009). Vivaty Create: What Developers Want. Consultado el 15 de 02 de 2009. En: http://www.vivaty.com/index.php

[49] W3c. (2007). ¿Qué es el Modelo de Objetos del Documento? Consultado el 14 de 5 de 2009. En: http://www.w3.org/2005/03/DOM3Core-es/

[50] W3c. (2009). Cascading Style Sheets Learning CSS. Consultado el 20 de 4 de 2009. En: http://www.w3.org/Style/

[51] Web3D Consortium. (2008). X3D Scene access interface Edition 2. Consultado el 17 de 2 de 2009. En: http://www.web3d.org/x3d/specifications/

[52] Web3D, C. (2009). Tools Web3D. Consultado el 10 de 2 de 2009. En: http://www. web3d.org/

[53] Web3D, C. (2008). X3D Architecture and base components. Consultado el 3 de 3 de 2009. En: http://www.web3d.org/x3d/specifications/

[54] Wikipedia. (2009). Psicomotricidad. Consultado el 19 de 2 de 2009. En: http:// es.wikipedia.org/wiki/Psicomotriz. 